\title{
Lead Selenide Nanostructures Self-Assembled across Multiple Length Scales and Dimensions
}

\author{
Evan K. Wujcik, ${ }^{1}$ Stephanie R. Aceto, ${ }^{2}$ Radha Narayanan, ${ }^{3}$ and Arijit Bose ${ }^{2}$ \\ ${ }^{1}$ Materials Engineering And Nanosensor (MEAN) Laboratory, Dan F. Smith Department of Chemical Engineering, \\ Lamar University, P.O. Box 10053, Beaumont, TX, USA \\ ${ }^{2}$ Laboratory of Soft Colloids \& Interfaces, Department of Chemical Engineering, The University of Rhode Island, \\ Kingston, RI 02881, USA \\ ${ }^{3}$ Department of Chemistry, The University of Rhode Island, Kingston, RI 02881, USA
}

Correspondence should be addressed to Evan K. Wujcik; evan.wujcik@lamar.edu and Arijit Bose; bosea@egr.uri.edu

Received 12 June 2016; Revised 25 October 2016; Accepted 3 November 2016

Academic Editor: Thierry Baron

Copyright (C) 2016 Evan K. Wujcik et al. This is an open access article distributed under the Creative Commons Attribution License, which permits unrestricted use, distribution, and reproduction in any medium, provided the original work is properly cited.

A self-assembly approach to lead selenide ( $\mathrm{PbSe}$ ) structures that have organized across multiple length scales and multiple dimensions has been achieved. These structures consist of angstrom-scale 0D PbSe crystals, synthesized via a hot solution process, which have stacked into 1D nanorods via aligned dipoles. These 1D nanorods have arranged into nanoscale 2D sheets via directional shortranged attraction. The nanoscale 2D sheets then further aligned into larger 2D microscale planes. In this study, the authors have characterized the PbSe structures via normal and cryo-TEM and EDX showing that this multiscale multidimensional self-assembled alignment is not due to drying effects. These PbSe structures hold promise for applications in advanced materials-particularly electronic technologies, where alignment can aid in device performance.

\section{Introduction}

The interest in nanoscale materials stems from the fact that their properties (electrical, optical, chemical, and mechanical, among others) are a function of their size, chemical makeup, and underlying structure. Oriented two-dimensional (2D) materials $[1,2]$, tunable nanostructures [3-8], and quantum dots [9-11] have gained much attention in recent years due to their promising applications in a number of fields including energy [12], sensors [13, 14], and biomedicine [15]. Functional and multifunctional semiconducting nanoparticles are of considerable interest, as the bandgaps of these materials are tunable to a wide range of absorbances and emissions. Narrow-bandgap IV-VI semiconducting lead selenide $(\mathrm{PbSe})$ structures have shown particular promise in third-generation solar cells, infrared optoelectronics, low cost microelectronics, and biological imaging and biosensor systems $[16,17]$. Hence, recent work on the colloidal and composite synthesis, fabrication, and processing of $\mathrm{PbSe}$ structures has gained interest among a wide range of disciplines.
In this work, we present a PbSe structure self-assembled system where the structures have aligned across multiple length scales and dimensions (Figure 1), where the term selfassembly refers to the autonomous organization of components into patterns or structures without human intervention [18]. Although self-assembly originated in the study of molecules, it is a strategy that is applicable at all scales [18], as shown through this work. These structures consist of angstrom-scale $0 \mathrm{D} \mathrm{PbSe}$ crystals, which have stacked into $1 \mathrm{D}$ nanorods. These $1 \mathrm{D}$ nanorods have arranged into $2 \mathrm{D}$ nanosheets that further aligned into larger $2 \mathrm{D}$ microscale planes. These 2D structures differ from randomly distributed nanorods, nanosheets, nanocrystals, and so forth and may hold promise due to their potentially enhanced properties. These oriented structures could easily be implemented into photovoltaic devices for increased efficiency-via their multiple exciton generation [19-23]. PbSe structures, such as these, are also of special interest for electrical applications due to their easily controlled alignment and connectivity [24]. 


\section{Materials and Methods}

Tri-n-octylphosphine (TOP), selenium powder, lead acetate trihydrate, oleic acid, n-tetradecylphosphonic acid, and diphenyl ether were purchased from Sigma-Aldrich for the synthesis. All syntheses were done under dry nitrogen. The synthesis of the PbSe structures followed an existing hot solution synthesis route [25] with modifications. A $1.0 \mathrm{M}$ stock solution of TOPSe was prepared by adding $7.86 \mathrm{~g}$ of selenium to $100 \mathrm{~mL}$ of TOP and mixing for 2 hours at $50^{\circ} \mathrm{C}$. Lead oleate was formed in situ by mixing $0.76 \mathrm{~g}$ of lead acetate trihydrate with $2 \mathrm{~mL}$ of oleic acid in $10 \mathrm{~mL}$ diphenyl ether and heating for 30 minutes at $150^{\circ} \mathrm{C}$ under nitrogen flow via a bubbler. The lead oleate solution was then cooled to $60^{\circ} \mathrm{C}$ and $4 \mathrm{~mL}$ of TOPSe was added to this solution (lead oleateTOPSe). In a separate beaker, $0.2 \mathrm{~g}$ of $\mathrm{n}$-tetradecylphosphonic acid was added to $15 \mathrm{~mL}$ of diphenyl ether and heated to $250^{\circ} \mathrm{C}$ with vigorous stirring. The lead oleate-TOPSe solution was then added to the solution of n-tetradecylphosphonic acid in diphenyl ether. The final solution was heated for 50 seconds at $250^{\circ} \mathrm{C}$ and then cooled to room temperature slowly. While the solution cools, the liquid turns cloudy, indicating the formation of the PbSe structures. Finally, $31 \mathrm{~mL}$ of hexane is added to this solution. These PbSe structures were then centrifuged and resuspended in different solvents (chloroform, water, and tetrahydrofuran). These are compared to the same structures with the capping agent (TOP) removed by a heat treatment [26], where the nanoparticles are heat-treated at $400^{\circ} \mathrm{C}$ in air for one hour. The procedures followed were in accordance with the ethical standards of the institutions involved.

Normal and cryogenic transmission electron microscopy (TEM) and energy dispersive X-ray spectroscopy (EDX) of the PbSe structures were conducted on a JEOL JEM 2100 instrument operated at $200 \mathrm{keV}$. Sample preparation for cryoTEM was done using a Vitrobot automated vitrification robot on lacey-carbon grids. Normal TEM samples were prepared on carbon-formvar 300-mesh copper grids. Here, an aliquot of $\mathrm{PbSe}$ in hexane was pipetted onto a grid and allowed to dry in open air.

\section{Results and Discussion}

3.1. Transmission Electron Microscopy. The TEM micrographs of the PbSe structures with TOP, shown in Figure 2, show the alignment of the individual $2 \mathrm{D}$ sheets, as well as the bulk arrangement of the structure into a microscale 2D plane. In Figure 2(a), the individual structures, made up of stacked $\mathrm{PbSe}$ nanorods, seem to be further aligned in bulk. It can be observed that this bulk arrangement seems to also align in a particularly ordered pattern, which could serve as an easily distributed array of further self-assembled structures. Figure 2(b) shows a higher magnified micrograph in which the individual structures, which make up the sheets, can be seen. The average length of the structures is distributed in a narrow range of $100 \mathrm{~nm}-150 \mathrm{~nm}$, which can also be seen in Figure 2(c). The evenly spaced aligned nanorods can be seen in Figure 2(d), where the individual structures are made up of many much smaller nanorods. Previous results [25] show that

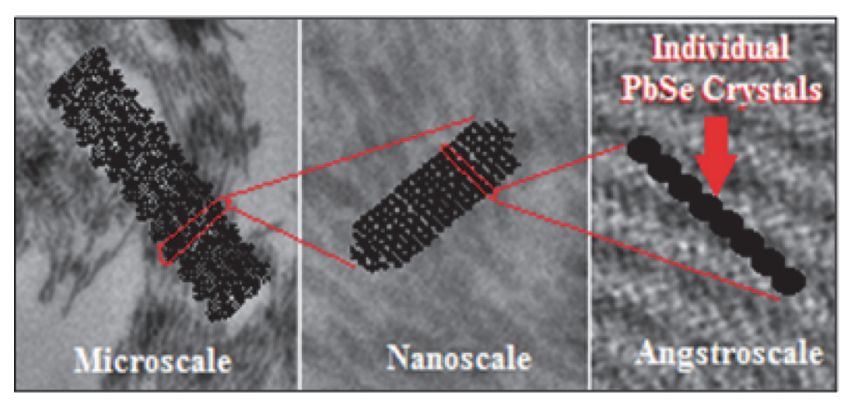

FIgURE 1: Schematic representation and illustrative TEM images (background) of PbSe structure alignment across multiple length scales and dimensions [2D planes (microscale) $\rightarrow 2 \mathrm{D}$ sheets (nanoscale) $\rightarrow 1 \mathrm{D}$ rods (nanoscale) $\rightarrow 0 \mathrm{D}$ crystals (angstrom-scale)].

the parameters controlling the formation in this synthesis route are the reaction temperature, reactant concentrations, and the concentration/composition of stabilizing agents, where the injection and growth temperatures show the greatest influence on the formation of the nanostructures. Figure 2(d) also shows some larger speckled dots, and, based on the EDX analysis, these dots can be attributed to undissolved or unreacted lead and selenium, as seen in other nanosemiconductor synthesis models [27].

The possibility that this stacking was being caused by drying of the samples on the grid was examined using cryogenic transmission electron microscopy. Stacked structures are seen in Figures 3(a) and 3(b), which proves that the alignment of the structures is not due to drying and exists in solution. Figure 3(b) is a high-magnification micrograph that shows that the nanorods, in terms of both spacing and size distribution, are uniform with an average of $1.8 \pm$ $0.2 \mathrm{~nm}$ in diameter and an average center-to-center spacing of $4.0 \pm 0.2 \mathrm{~nm}$. Measurements were made using free ImageJ open-source software (available on the NIH website: http://rsbweb.nih.gov/ij/).

3.2. Energy Dispersive X-Ray Spectroscopy. The energy dispersive X-ray spectroscopy (EDX) analysis is shown in Figure 4. The PbSe structures exhibit both major and minor peaks of lead $(\mathrm{L} \alpha=10.5 \mathrm{keV}, \mathrm{M} \alpha=2.3 \mathrm{keV})$ and selenium $(\mathrm{K} \alpha=$ $11.2 \mathrm{keV}, \mathrm{L} \alpha=1.3 \mathrm{keV}$ ). This is characteristic of the presence of $\mathrm{PbSe}$. The copper, carbon, and oxygen peaks can be attributed to the carbon-formvar copper TEM grid.

$\mathrm{PbSe}$ structures that have aligned across multiple length scales and dimensions were synthesized and characterized through TEM and EDX. Given their nanoscale size and reported multiscale multidimensional orientation, these $\mathrm{PbSe}$ structures can serve as inorganic semiconductors for advanced materials. This differs from typical PbSe structures, which exhibit absorption over a wide range of the infrared spectrum [28] where spectral irradiance is sparse and is divided up by many $\mathrm{H}_{2} \mathrm{O}$ and $\mathrm{CO}_{2}$ absorption bands. Although self-assembly originated in the study of molecules, it is a strategy that is applicable at all scales [18], as shown through this work. 


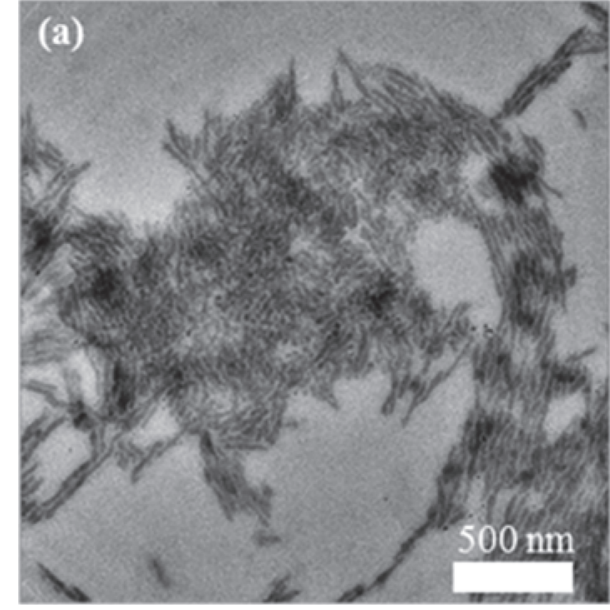

(a)

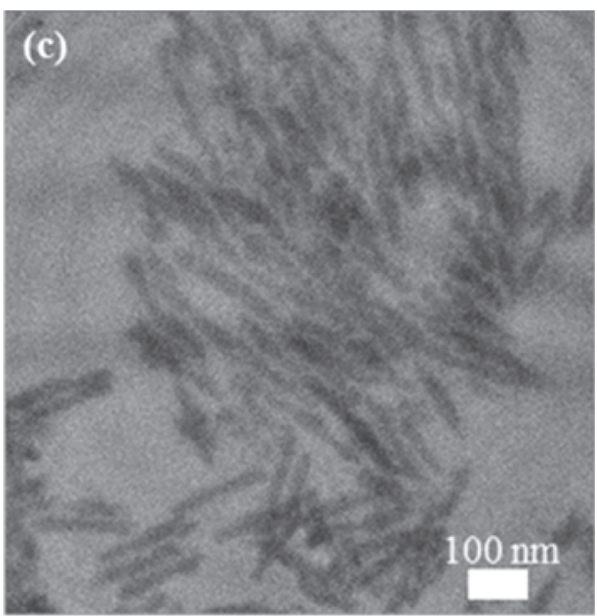

(c)

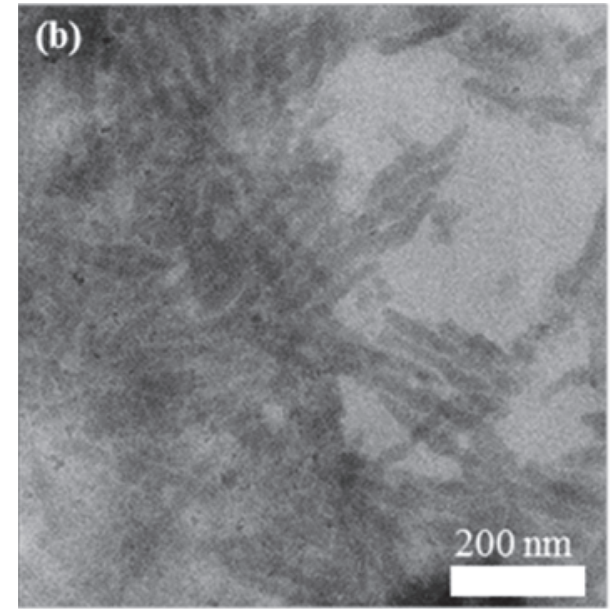

(b)

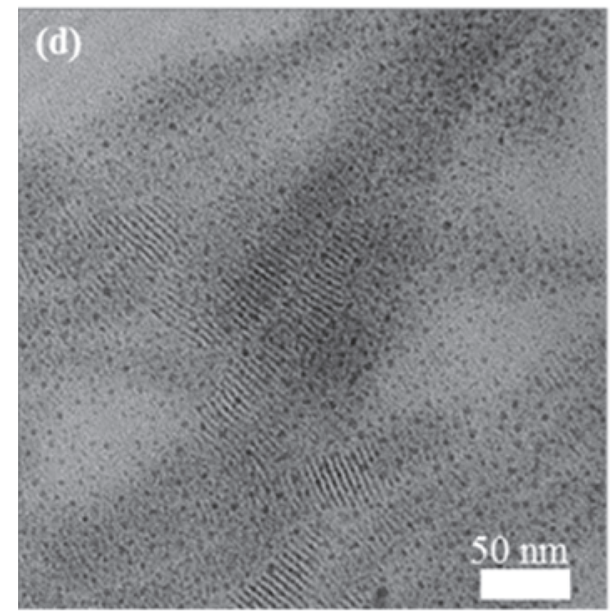

(d)

FIGURE 2: TEM micrographs showing the (a-b) ordered bulk alignment of PbSe structures, (c) the average length of the structures distributed in a narrow range of $100 \mathrm{~nm}-150 \mathrm{~nm}$, and (d) individual structures, which are made up of many much smaller nanorods.

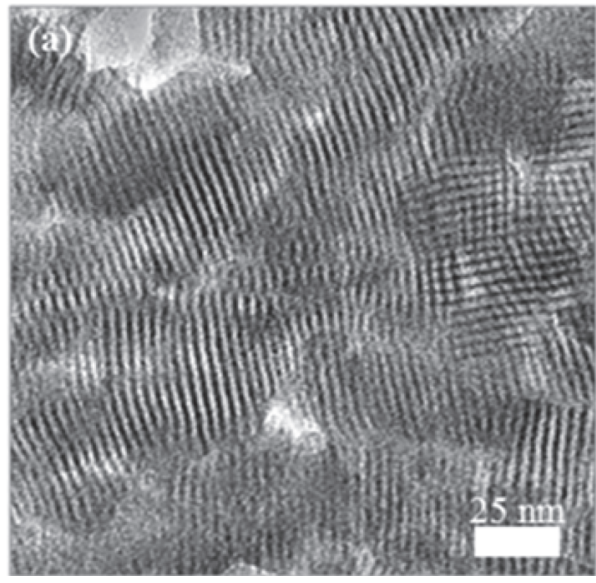

(a)

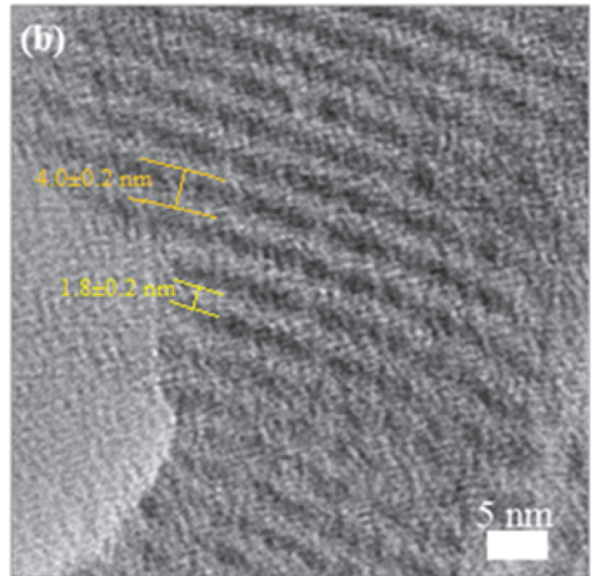

(b)

FIGURE 3: (a) A cryo-TEM micrograph showing the structure of the aligned PbSe NRs with TOP and a (b) cryo-TEM micrograph which shows the aligned nanorods also with TOP, which are on average $1.8 \pm 0.2 \mathrm{~nm}$ in diameter with an average center-to-center spacing of $4.0 \pm 0.2 \mathrm{~nm}$. 


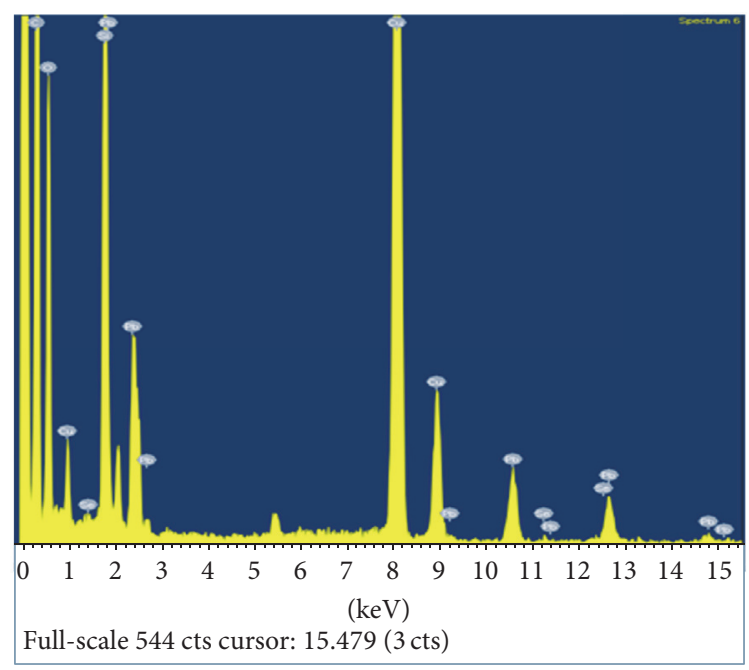

FIGURE 4: EDX analysis of the PbSe structures, showing both major and minor peaks of lead ( $\mathrm{L} \alpha=10.5 \mathrm{keV}, \mathrm{M} \alpha=2.3 \mathrm{keV})$ and selenium $(\mathrm{K} \alpha=11.2 \mathrm{keV}, \mathrm{L} \alpha=1.3 \mathrm{keV})$.

The observed system is an example of static self-assembly-one in which the system is at global or local equilibrium and does not dissipate energy [18]. One-dimensional nanostructure growth has been studied for a number of years, having a number of assembly mechanisms [29-31]. Dipole-dipole interactions are the most likely candidate for the driving force directing the $0 \mathrm{D}$ PbSe crystals to $1 \mathrm{D}$ rod assembly mechanism $[25,32]$, reinforced by the isotropy of the crystals-each having six identical $\{100\}$ facets. Previous models have also shown that this system should have permanent nonzero dipole moments-up to $89 \%$-with a prediction of the largest dipole moment along the $\langle 100\rangle$ axis [25]. Similar stacking of $1 \mathrm{D}$ rods to $2 \mathrm{D}$ sheets has been shown in other systems [33], where the interfacial interactions play a key role in the $2 \mathrm{D}$ alignment. Similar mechanisms are suspected to be at play in the current system; however, further investigation is needed. If the system is found to unalign upon removal of TOP, then it is possible that alignment is due to directional short-ranged attraction or molecular self-assembly, such as hydrogen and coordination bonding, Van der Waals interactions, electrostatic interactions, or hydrophobic interactions.

TEM of the TOP-removed structures has yet to be completed but is expected to yield unaligned rods. If this hypothesis is consistent with the results obtained, it could be speculated that the structures are aligning due to enthalpic or entropic effects between the hydrophobic chains of the capping agent, TOP, or neighboring structures. This case would be an example of meso- or macroscopic interactions (i.e., gravitational interactions and external electromagnetic, capillary, and entropic interactions). This has been observed as 1and 2-dimensional structures in some nanoparticle systems of differing shapes and sizes [34-36] and has been attributed to the combination of size, directional attraction, and hydrophobic and electrostatic interactions of the system [37].

Further experimentation to be investigated, with the confirmation that stacking continues after the removal of
TOP, could include a magnetic or polarization study to see whether the alignment is due to a dipole in the PbSe structures. Magnetically and photomagnetically forced alignment and positioning have been displayed in a number of quantum dot and nanorod anisotropic electrostatic systems [38-43]. However, all are induced by taking advantage of dipolar interactions upon applying a magnetic force and/or doping the nanocrystals with a magnetically sensitive cation; therefore, these systems do not spontaneously self-assemble. The authors also do not suspect any influence of the carbonformvar copper TEM grid on the assembly.

\section{Conclusions}

In conclusion, the presented PbSe self-assembled systemwhere the structures have aligned across multiple length scales and dimensions - has been characterized and explored. It has been shown that these structures consist of angstromscale $0 \mathrm{D}$ PbSe crystals, which have stacked into $1 \mathrm{D}$ nanorods and then are further arranged into $2 \mathrm{D}$ nanosheets. These $2 \mathrm{D}$ nanosheets then align into larger 2D microscale planes. Self-assembled ensembles such as these provide a solution to the fabrication of ordered aggregates from components with sizes ranging from the angstrom to micron scale. The interest here lies in the notion that these components typically are found between the sizes that can be manipulated by chemistry and those that can be manipulated by conventional macro techniques. This range of sizes is extremely important for the further development of nanotechnology in the areas of inorganic or biomimetic syntheses and requires further investigation as an entire field.

In continuing this work, thermal analysis will be conducted to further verify that the TOP has been removed. This will investigate the thermal properties of the $\mathrm{PbSe}$ structures as well, but it should be noted that-being entirely inorganic - any photodegradation of these structures would be insignificant compared to the materials used in common hybrid organic/inorganic systems. Future work will also focus on a tighter control of the alignment, size, and shape of the PbSe structures, as well as the determination of the assembly mechanism, further advanced characterization, and integration into devices. In electronics, namely, nanoelectronics, where the importance of single-particle interactions is magnified, alignment and symmetry are of the utmost importance, making this unique self-assembled alignment advantageous for enhanced optical, magnetic, and/or electrical properties, particularly in advanced materials.

\section{Competing Interests}

The authors declare that they have no competing interests.

\section{Acknowledgments}

The authors would like to acknowledge the NASA funded Rhode Island Space Grant Consortium, the University of Rhode Island Transportation Center, and the Honda Initiation Grant for their financial support. 


\section{References}

[1] M. P. Boneschanscher, W. H. Evers, J. J. Geuchies et al., "Longrange orientation and atomic attachment of nanocrystals in $2 \mathrm{D}$ honeycomb superlattices," Science, vol. 344, no. 6190, pp. 13771380, 2014.

[2] C. Schliehe, B. H. Juarez, M. Pelletier et al., "Ultrathin PbS sheets by two-dimensional oriented attachment," Science, vol. 329, no. 5991, pp. 550-553, 2010.

[3] X. Liu, J. Wan, Y. Xiong, S. Liang, Y. Gao, and Z. Tang, "Synthesis of uniform CdSe quantum wires via oriented attachment," Journal of Nanoscience and Nanotechnology, vol. 15, no. 8, pp. 5798-5806, 2015.

[4] G. Shao, G. Chen, W. Yang, T. Ding, J. Zuo, and Q. Yang, "Organometallic-route synthesis, controllable growth, mechanism investigation, and surface feature of PbSe nanostructures with tunable shapes," Langmuir, vol. 30, no. 10, pp. 2863-2872, 2014.

[5] K. A. Fichthorn, "Atomic-scale aspects of oriented attachment," Chemical Engineering Science, vol. 121, pp. 10-15, 2015.

[6] A. Sobhani, F. Davar, and M. Salavati-Niasari, "Synthesis and characterization of hexagonal nano-sized nickel selenide by simple hydrothermal method assisted by CTAB," Applied Surface Science, vol. 257, no. 18, pp. 7982-7987, 2011.

[7] A. Sobhani, M. Salavati-Niasari, and F. Davar, "Shape control of nickel selenides synthesized by a simple hydrothermal reduction process," Polyhedron, vol. 31, no. 1, pp. 210-216, 2012.

[8] M. Esmaeili-Zare, M. Salavati-Niasari, and A. Sobhani, "Simple sonochemical synthesis and characterization of HgSe nanoparticles," Ultrasonics Sonochemistry, vol. 19, no. 5, pp. 1079-1086, 2012.

[9] K. Whitham, J. Yang, B. H. Savitzky, L. F. Kourkoutis, F. Wise, and T. Hanrath, "Charge transport and localization in atomically coherent quantum dot solids," Nature Materials, vol. 15, pp. 557-563, 2016.

[10] S. M. Islam and P. Banerji, "Size effect of InAs quantum dots grown by metal organic chemical vapor deposition technique in storing electrical charges for memory applications," RSC Advances, vol. 5, no. 9, pp. 6906-6911, 2015.

[11] Q. Wu, L. Chen, L. Huang et al., "Quantum dots decorated gold nanorod as fluorescent-plasmonic dual-modal contrasts agent for cancer imaging," Biosensors and Bioelectronics, vol. 74, pp. 16-23, 2015.

[12] Y. Wang, H. Wei, Y. Lu, S. Wei, E. K. Wujcik, and Z. Guo, "Multifunctional carbon nanostructures for advanced energy storage applications," Nanomaterials, vol. 5, no. 2, pp. 755-777, 2015.

[13] I. Afreh, E. K. Wujcik, N. Blasdel et al., "Detection of halogenated organics by their inhibitory action in a catalytic reaction between dimethyl acetylenedicarboxylate and 2-methyl-4nitroaniline," Journal of Analytical Chemistry, vol. 70, no. 7, pp. 825-830, 2015.

[14] E. K. Wujcik, N. J. Londoño, S. E. Duirk, C. N. Monty, and R. I. Masel, "An acetylcholinesterase-inspired biomimetic toxicity sensor," Chemosphere, vol. 91, no. 8, pp. 1176-1182, 2013.

[15] N. J. Blasdel, E. K. Wujcik, J. E. Carletta, K.-S. Lee, and C. N. Monty, "Fabric nanocomposite resistance temperature detector," IEEE Sensors Journal, vol. 15, no. 1, pp. 300-306, 2015.

[16] S. V. Kershaw, A. S. Susha, and A. L. Rogach, "Narrow bandgap colloidal metal chalcogenide quantum dots: synthetic methods, heterostructures, assemblies, electronic and infrared optical properties," Chemical Society Reviews, vol. 42, no. 7, pp. $3033-$ 3087, 2013.

[17] M. C. Beard, J. M. Luther, O. E. Semonin, and A. J. Nozik, “Third generation photovoltaics based on multiple exciton generation in quantum confined semiconductors," Accounts of Chemical Research, vol. 46, no. 6, pp. 1252-1260, 2013.

[18] G. M. Whitesides and B. Grzybowski, "Self-assembly at all scales," Science, vol. 295, no. 5564, pp. 2418-2421, 2002.

[19] R. D. Schaller and V. I. Klimov, "High efficiency carrier multiplication in PbSe nanocrystals: implications for solar energy conversion," Physical Review Letters, vol. 92, no. 18, Article ID 186601, 2004.

[20] R. J. Ellingson, M. C. Beard, J. C. Johnson et al., "Highly efficient multiple exciton generation in colloidal $\mathrm{PbSe}$ and $\mathrm{PbS}$ quantum dots," Nano Letters, vol. 5, no. 5, pp. 865-871, 2005.

[21] S. J. Kim, W. J. Kim, Y. Sahoo, A. N. Cartwright, and P. N. Prasad, "Multiple exciton generation and electrical extraction from a PbSe quantum dot photoconductor," Applied Physics Letters, vol. 92, no. 3, Article ID 031107, 2008.

[22] M. C. Beard, A. G. Midgett, M. Law, O. E. Semonin, R. J. Ellingson, and A. J. Nozik, "Variations in the quantum efficiency of multiple exciton generation for a series of chemically treated PbSe nanocrystal films," Nano Letters, vol. 9, no. 2, pp. 836-845, 2009.

[23] R. D. Schaller, M. Sykora, J. M. Pietryga, and V. I. Klimov, "Seven excitons at a cost of one: redefining the limits for conversion efficiency of photons into charge carriers," Nano Letters, vol. 6, no. 3, pp. 424-429, 2006.

[24] D. V. Talapin, C. T. Black, C. R. Kagan, E. V. Shevchenko, A. Afzali, and C. B. Murray, "Alignment, electronic properties, doping, and on-chip growth of colloidal PbSe nanowires," Journal of Physical Chemistry C, vol. 111, no. 35, pp. 13244-13249, 2007.

[25] K.-S. Cho, D. V. Talapin, W. Gaschler, and C. B. Murray, "Designing PbSe nanowires and nanorings through oriented attachment of nanoparticles," Journal of the American Chemical Society, vol. 127, no. 19, pp. 7140-7147, 2005.

[26] A. P. Caricato, M. Epifani, M. Martino et al., "MAPLE deposition and characterization of $\mathrm{SnO}_{2}$ colloidal nanoparticle thin films," Journal of Physics D: Applied Physics, vol. 42, no. 9, Article ID 095105, 2009.

[27] A. Khanaki, H. Abdizadeh, and M. R. Golobostanfard, "Effects of process parameters on the synthesis and characterization of $\mathrm{CuIn}_{1-x} \mathrm{Ga}_{x} \mathrm{Se}_{2}$ nanopowders produced by new modified solvothermal method," Materials Science in Semiconductor Processing, vol. 16, no. 6, pp. 1397-1404, 2013.

[28] H. Du, C. Chen, R. Krishnan et al., "Optical properties of colloidal PbSe nanocrystals," Nano Letters, vol. 2, no. 11, pp. 1321-1324, 2002.

[29] J. Y. Cheng, F. Zhang, V. P. Chuang, A. M. Mayes, and C. A. Ross, "Self-assembled one-dimensional nanostructure arrays," Nano Letters, vol. 6, no. 9, pp. 2099-2103, 2006.

[30] M. Sam, N. Moghimian, and R. B. Bhiladvala, "Field-directed chaining of nanowires: towards transparent electrodes," Materials Letters, vol. 163, pp. 205-208, 2016.

[31] P. Kumar, R. Kumar, and H.-N. Lee, "Magnetic field induced one-dimensional nano/micro structures growth on the surface of iron oxide thin film," Thin Solid Films, vol. 592, pp. 155-161, 2015.

[32] M. Shim and P. Guyot-Sionnest, "Permanent dipole moment and charges in colloidal semiconductor quantum dots," The Journal of Chemical Physics, vol. 111, no. 15, pp. 6955-6964, 1999. 
[33] W. Cheng, Y. Ju, P. Payamyar et al., "Large-area alignment of Tungsten oxide nanowires over flat and patterned substrates for room-temperature gas sensing," Angewandte ChemieInternational Edition, vol. 54, no. 1, pp. 340-344, 2015.

[34] Z. Tang, Z. Zhang, Y. Wang, S. C. Glotzer, and N. A. Kotov, "Self-assembly of CdTe nanocrystals into free-floating sheets," Science, vol. 314, no. 5797, pp. 274-278, 2006.

[35] Z. Zhang and S. C. Glotzer, "Self-assembly of patchy particles," Nano Letters, vol. 4, no. 8, pp. 1407-1413, 2004.

[36] R. P. Carney, G. A. DeVries, C. Dubois et al., "Size limitations for the formation of ordered striped nanoparticles," Journal of the American Chemical Society, vol. 130, no. 3, pp. 798-799, 2008.

[37] Z. Zhang, Z. Tang, N. A. Kotov, and S. C. Glotzer, "Simulations and analysis of self-assembly of CdTe nanoparticles into wires and sheets," Nano Letters, vol. 7, no. 6, pp. 1670-1675, 2007.

[38] A. Zakrassov, A. Bitler, L. Etgar, G. Leitus, E. Lifshitz, and R. Naaman, "Controlling the anisotropic magnetic dipolar interactions of PbSe self-assembled nanoparticles on GaAs," Physical Chemistry Chemical Physics, vol. 11, no. 35, pp. 75497552, 2009.

[39] M. Tanase, L. A. Bauer, A. Hultgren et al., "Magnetic alignment of fluorescent nanowires," Nano Letters, vol. 1, no. 3, pp. 155-158, 2001.

[40] S.-W. Lee, M.-C. Jeong, J.-M. Myoung, G.-S. Chae, and I.-J. Chung, "Magnetic alignment of $\mathrm{ZnO}$ nanowires for optoelectronic device applications," Applied Physics Letters, vol. 90, no. 13, Article ID 133115, 2007.

[41] R. Beaulac, L. Schneider, P. I. Archer, G. Bacher, and D. R. Gamelin, "Light-induced spontaneous magnetization in doped colloidal quantum dots," Science, vol. 325, no. 5943, pp. 973-976, 2009.

[42] C. M. Hangarter and N. V. Myung, "Magnetic alignment of nanowires," Chemistry of Materials, vol. 17, no. 6, pp. 1320-1324, 2005.

[43] J. C. Love, A. R. Urbach, M. G. Prentiss, and G. M. Whitesides, "Three-dimensional self-assembly of metallic rods with submicron diameters using magnetic interactions," Journal of the American Chemical Society, vol. 125, no. 42, pp. 12696-12697, 2003. 

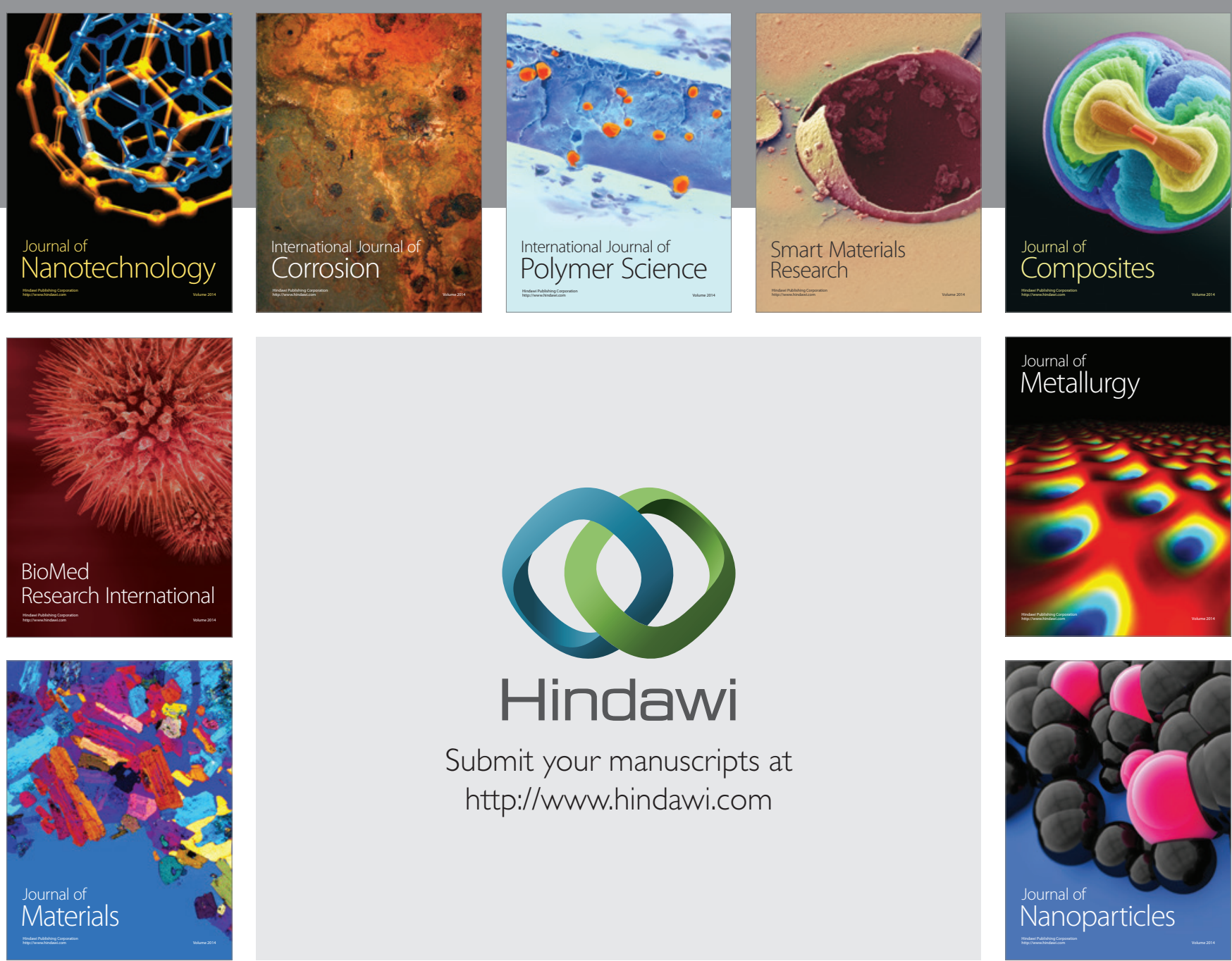

\section{Hindawi}

Submit your manuscripts at

http://www.hindawi.com

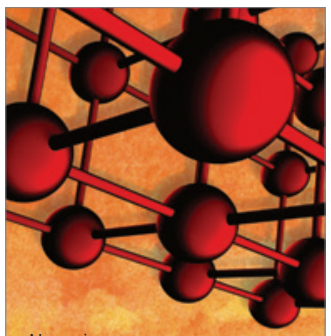

Materials Science and Engineering
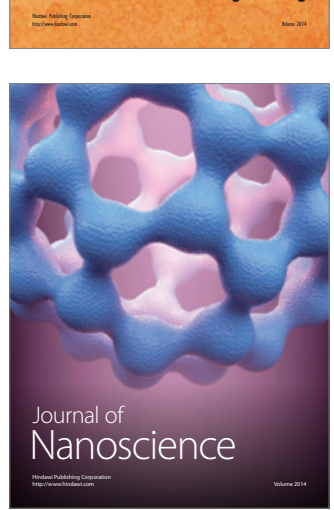
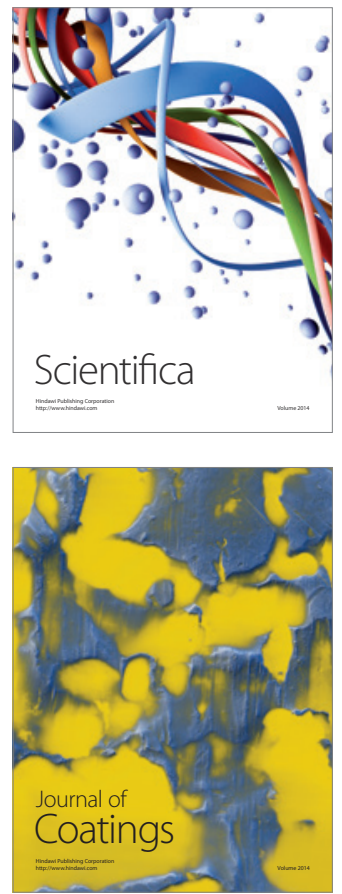
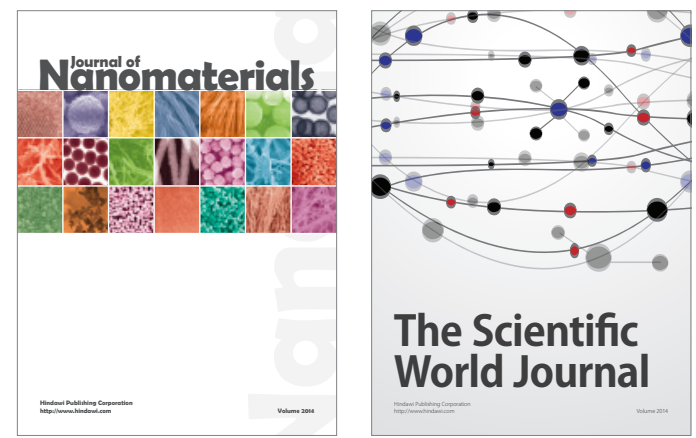

The Scientific World Journal
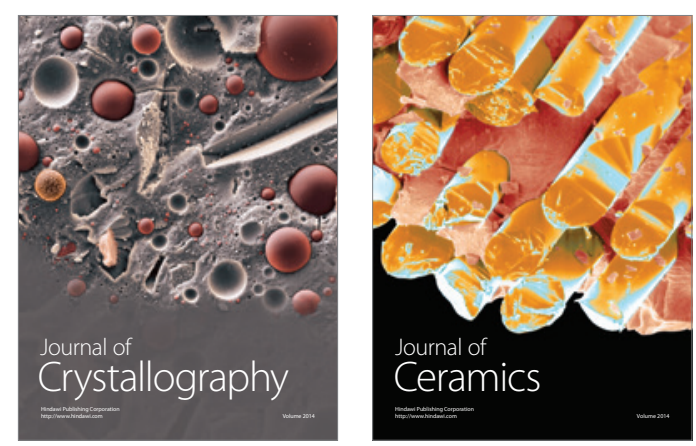
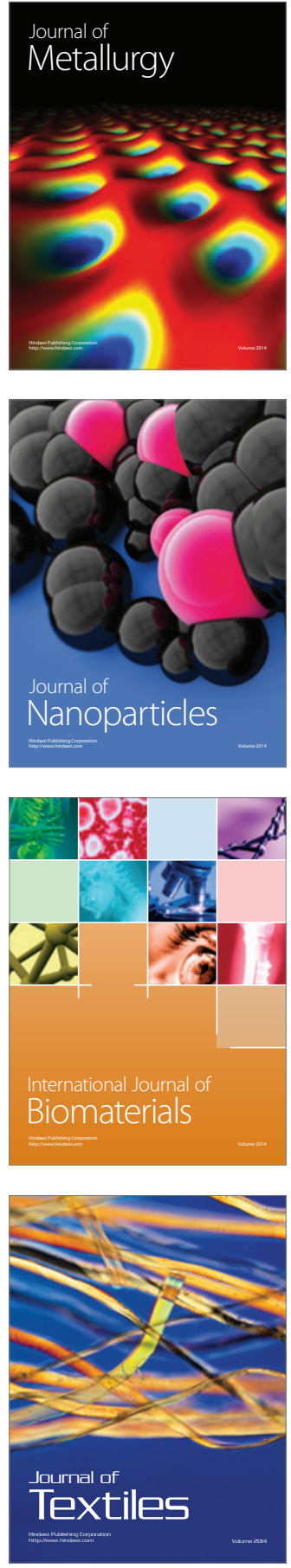